\title{
Los territorios de la formación para la investigación en biología
}

Miriam-Liset Flores y Aníbal-Roque Bar

\section{RESUMEN}

A través de una metodología de estudios de casos, nos propusimos reconstruir las distintas praxis que se expresan en los diferentes territorios formativos en biología, haciendo foco en la dimensión epistemológica de la disciplina. Como resultado reconocemos cuatro territorios: campo, laboratorio, escritorio y jornadas científicas. En los dos primeros se propicia la aproximación al objeto real y en los últimos se reconstruye al objeto de manera discursiva. Se advierte una dinámica dialéctica entre los territorios, evidenciando la recursividad del proceso de investigación más allá del discurso disciplinar presentado como neutro y lineal.

Palabras clave: formación de investigadores, universidad, biología, Argentina.

\section{Miriam-Liset Flores}

Argentina. Profesora en Ciencias de la Educación, Universidad Nacional del Nordeste, Argentina. Becaria Doctoral, Instituto de Investigaciones en Educación, Facultad de Humanidades, Universidad Nacional del Nordeste (UNNE)-CONICET, Argentina. Temas de investigación: formación disciplinar, formación para la investigación.

\section{Aníbal-Roque Bar}

anibalrbar@gmail.com

Argentino. Doctor en Ciencias Cognitivas, Universidad Nacional del Nordeste (UNNE), Argentina. Profesor Titular Regular, Facultad de Humanidades, UNNE, Argentina. Temas de investigación: formación disciplinar, formación para la investigación. 


\section{Os territórios da formação para a pesquisa em biologia}

\section{RESUMO}

Através de uma metodologia de estudos de casos, nos propusemos reconstruir as distintas praxis que se expressam nos diferentes territórios formativos em biologia, enfocando na dimensão epistemológica da disciplina. Como resultado reconhecemos quatro territórios: campo, laboratório, escritório e jornadas científicas. Nos dois primeiros se propicia a aproximação ao objeto real e nos últimos se remete ao objeto de maneira discursiva. Se adverte uma dinâmica dialética entre os territórios, evidenciando a recursividade do processo de pesquisa além do discurso disciplinar apresentado como neutro e linear.

Palavras chave: formação de pesquisadores, universidade, biologia.

\section{The Territories of Training for Research in Biology}

\section{ABSTRACT}

Through a methodology of case studies, the authors proposed to reconstruct the different praxis that are expressed in specific formative territories in Biology, focusing on the epistemological dimension of the discipline. As a result, the authors identify four territories: field, laboratory, desk and scientific conferences. In the first two the approach to the real object is propitiated and in the last two the object is reconstructed in a discursive way. What can be observed is a dialectic dynamic between the territories, evidencing the recursiveness of the research process beyond the disciplinary discourse presented as neutral and linear.

Key words: training of researchers, university, Biology. 


\section{Introducción}

El presente trabajo ${ }^{1}$ forma parte de un proyecto de investigación denominado "Formación para la investigación disciplinar en la universidad. Un estudio sobre contextos, sujetos y procesos cognitivos", desarrollado en la Universidad Nacional del Nordeste.

Específicamente, aquí abordamos la problemática de la formación para la investigación disciplinar en biología, atendiendo la incidencia de la dimensión epistemológica de la disciplina en los distintos territorios en los que se forman los integrantes de los equipos de investigación.

Hemos decidido recuperar los aportes de Echeverría (1998) quien describe cuatro instancias para dar cuenta de la dinámica tecnocientífica: el contexto de educación, entendiéndose como todas las acciones educativas que se desarrollan en formación inicial; el contexto de innovación y el contexto de evaluación, correspondientes a la producción y validación del conocimiento científico, y por último, el contexto de aplicación. ${ }^{2}$

De modo general, ubicamos en el primer contexto a los estudiantes y becarios de pregrado. En el segundo y tercero posicionamos a los becarios, a los investigadores en formación y a los directores de equipos.

Atendiendo a una caracterización específica de cada contexto, el primero de ellos se encuentra abocado a la enseñanza y a la difusión de la ciencia. Es aquí donde se inician los procesos científicos, ya que comprende dos acciones recíprocas fundamentales: la enseñanza y el aprendizaje de sistemas conceptuales y lingüísticos, por un lado, pero también de representaciones e imágenes científicas, técnicas operatorias, problemas y manejo de instrumentos, por otro.

Bar (2013) expresa que la comprensión de enunciados y textos disciplinares de contenido biológico suponen la apropiación de un sistema de complejos conocimientos teóricos y prácticos que habilitan luego la posibilidad de descubrir, justificar y aplicar el saber disciplinar. Tales aprendizajes configuran la matriz central sobre la cual se construirán otros procesos clave para su adscripción a determinadas comunidades científicas, que luego consolidarán la ontología de los procesos biológicos y las bases para el desarrollo de sus dispositivos metodológicos.

Considerando al contexto disciplinar de la biología, ésta se sustenta en principios monoparadigmáticos, centrada en un mismo cuerpo teórico de conocimiento, es fuertemente normativa, conservadora, y abocada al campo de la investigación (Biglan, 1973).

Bar (2013) concluye que la formación disciplinar en biología es un largo proceso que se inicia en la universidad, en el cual a los estudiantes se les brinda lo necesario para iniciar el camino profesional, se les marca un horizonte de posibilidad a través del desarrollo de contenidos y modos de operar con ellos, promoviendo de este modo la constitución de sujetos que puedan desempeñarse en el marco del paradigma. De este modo, la formación da condiciones para la comprensión de la ontología subyacente, ya que apunta a comprender qué son los fenómenos biológicos, profundizando la cuestión metodológica a posteriori cuando el estudiante comprende debidamente la naturaleza de los fenómenos de su disciplina.

En el contexto de educación no sólo se encuentran los estudiantes, sino también los becarios de pregrado, adscritos o pasantes de investigación, quienes paralelamente, además de la formación inicial, se hallan transitando los inicios en sus praxis investigativas, introduciéndose en los conocimientos conceptuales y metodológicos de una línea de investigación específica junto con un equipo de investigación consolidado.

\footnotetext{
${ }^{1}$ Agradecemos a los integrantes de los equipos de investigación, quienes nos aportaron su valiosa experiencia para el presente estudio.

${ }^{2}$ Para este trabajo sólo consideramos los tres primeros contextos, ya que el contexto aplicación no forma parte del objetivo de nuestro estudio.
} 
Cabe destacar que si bien becarios y tesistas suponen una experiencia más protagónica que la del estudiante en la producción de conocimiento disciplinar, su actividad se encuentra fuertemente normada, ya que responde a una lógica tutorial en la cual los márgenes de autonomía son estrechos. Sus propios proyectos se inscriben en líneas de investigación ya instituidas, gran parte de las decisiones respecto de sus planes de trabajo son dirimidas por sus directores, y sus acciones están reguladas por plazos preestablecidos por los organismos de evaluación y financiamiento de las actividades de ciencia y tecnología.

A modo de contextualización, en Argentina la labor investigativa se desarrolla a través de proyectos de investigación financiados por diferentes organismos. En términos generales, suelen estar integrados por un director con trayectoria académica, por integrantes que ya han transitado distintos trayectos y se encuentran en proceso de formación, y otros que inician en la investigación a través de becas $^{3}$ de pre y posgrado. Eventualmente en dicho equipo se desarrollan proyectos de investigación externos de otros sujetos que realizan sus tesis sin pertenecer formalmente al equipo.

En referencia a los equipos de investigación, considerando que sus integrantes (becarios de pre y posgrado, investigadores en formación y los investigadores consolidados) se encuentran inmersos en la lógica de los procesos científicos, sus praxis tendrían lugar fundamentalmente en los dos contextos restantes: innovación y evaluación de los conocimientos. El primero de ellos, entendido como la producción de conocimiento teórico, empírico, informativo y técnico; y el segundo ligado con una buena fundamentación metodológica y racional de la ciencia, adquiriendo principal protagonismo la comunidad científica, dado que es ella quien acepta nuevas hipótesis, teorías, problemas, descubrimientos e innovaciones.

Cortina (2003), afirma que la presencia de un experto en una actividad, en este caso la investigación, le dota de una peculiar identidad y genera un sentido de pertenencia en una comunidad, la que comparte tanto metas como formas de proceder ante el saber disciplinar y sus metodologías, y en las relaciones con los otros. En el mismo sentido, Fourtor Olliver (2011) expresa que los grupos operan como espacios donde, a la par de hacer investigación, los sujetos se van formando en una nueva metodología, ante un contenido disciplinar visto desde otras aristas u otros referentes teóricos, ante acciones más complejas de recolección o análisis de información, ante la escritura de distintos textos académicos o la presentación de resultados en distintos foros.

Un grupo puede funcionar como metodología para formar investigadores si el experto combina saberes prácticos, teóricos y pedagógicos. A todo ello, el autor citado con anterioridad agrega que el ethos profesional se trasmite en el contacto directo con los investigadores, en las tareas concretas realizadas en pos de la investigación, en las discusiones/debates en los que participan, en las explicaciones sobre cuestiones de orden epistémico, disciplinar, metodológico u operativo. De este modo, la formación en investigación es un proceso situado, que se da en determinados espacios, temporalidades y con ciertas personas, agregándole a todo ello un componente artesanal y singular.

Flores y Bar (2017), conceptualizaron incipientemente los territorios formativos para la investigación, entendiéndolo como la delimitación del espacio físico en el cual acontecen las interacciones

\footnotetext{
${ }^{3}$ En el contexto de la universidad pública argentina, los estudiantes avanzados y egresados de las diferentes carreras de grado pueden presentarse a convocatorias anuales para el otorgamiento de becas de investigación. Éstas son financiadas por diferentes organismos estatales de ciencia y tecnología: Secretaría General de Ciencia y Técnica, dependiente de la universidad (SGCyT), Consejo Nacional de Investigaciones Científicas y Técnicas (CONICET), Agencia Nacional de Promoción Científica y Tecnológica, entre otros. Quienes acceden a este tipo de becas, planifican y desarrollan proyectos bajo la orientación de docentes investigadores con una prolongada trayectoria en sus respectivos campos disciplinares.
} 
entre investigadores en diferentes instancias de formación, en un lapso de tiempo definido, en el cual se habilitan relaciones, operaciones cognitivas y físicas, que suponen también un vínculo de acercamiento con el objeto de estudio de la disciplina, y desde allí el juego con el conocimiento. En otras palabras, es el "locus" en el que tienen lugar los distintos procesos de la formación para la investigación y en donde se cristalizan las praxis formativas y epistemológicas de un modo específico y situado.

En función de lo expuesto, asumimos el supuesto de que la disciplina configurará las praxis formativas de investigación en términos de las dimensiones ontológicas epistemológicas y metodológicas. En este sentido, si bien la biología presenta una ontología realista, la mirada epistemológica será distinta en cada territorio formativo, ya que éste habilitaría un modo de acercamiento particular al objeto. Así, el objetivo del presente artículo es reconstruir las distintas praxisformativo disciplinares que se expresan en los diferentes territorios formativos en biología, haciendo foco en la dimensión epistemológica de la disciplina.

\section{Decisiones metodológicas}

Para el desarrollo del trabajo, nos posicionamos en una metodología de corte cualitativo, específicamente en los estudios de caso. Para Hartley (1994), lo que define el estudio de casos no es la técnica sino la orientación teórica puesta en la dinámica entre procesos y contextos, metodología que parece más apropiada para el análisis de los fenómenos sociales, donde la interpretación y la mirada holística muestran pertinencia (Stake, 1995).

En este sentido, los casos seleccionados para el presente estudio fueron dos equipos de investigación de la carrera de Biología de una universidad pública argentina, pertenecientes a dos subdisciplinas: Artrópodología y Paleontología, las cuales han sido escogidas en función de que tuvieron proyectos acreditados por organismos científicos en los últimos diez años, que evidenciaron producción académica en los últimos cinco años, y que formaron cuadros de investigadores.

En cuanto al caso de Artropodología, se trata de un equipo con gran trayectoria en docencia, investigación y extensión. Se halla estructurado en una cátedra que se encuentra en vínculo con diversas líneas de investigación en entomología (coleópteros, dípteros, formícidos) y aracnología. Se encuentra integrado por dos directoras, un investigador en formación, varios becarios de posgrado y pasantes en investigación. Cabe destacar que los tres primeros revistan como docentes con dedicación exclusiva en la facultad en la que se inserta la cátedra.

En el caso de Paleontología, se trata de un equipo que desarrolla docencia en la carrera de Biología, pero que sus actividades referentes a investigación se sustancian en un instituto del Consejo Nacional de Investigaciones Científicas y Técnicas (CONICET). El grupo se halla constituido por investigadores de CONICET y por docentes de la universidad con sede en el instituto, que operan como directores de sub-equipos alineados en paleobotánica, paleovertebrados, paleoinvertebrados (entre otras subdisciplinas), un gran número de investigadores en formación, numerosos becarios de pos y pregrado, y pasantes de investigación. La mayoría de ellos, con dedicación exclusiva en investigación.

Para la recolección de la información, se utilizaron entrevistas en profundidad, las que indagaron sobre los siguientes aspectos:

- Orientaciones en la construcción del objeto de investigación. Valor asignado a la observación, experimentación o cualquier otra instancia de objetivación del conocimiento. Valoración dada a la reflexión.

- Funciones de la especulación y la imaginación.

- Papel asignado a la bibliografia y a la producción de otros investigadores (marco referencial discutible, valor de autoridad, fundamento justificatorio de las conclusiones). 
- Concepción de descubrimiento (novedad que rompe con los cánones; novedoso pero asimilado a lo validado por la comunidad; más que novedad, instaura reformulaciones o reestructuraciones del campo; más que conceptos novedosos aporta nuevos casos de la teoría).

- Concepción de justificación (empírica, de orden lógico, mixta, pragmática).

Los informantes entrevistados en cada equipo de investigación fueron: ${ }^{4}$

Directores de equipo de investigación: aquellos que en los últimos diez años tuvieran proyectos de investigación acreditados, que fueran reconocidos por sus pares como investigadores plenamente formados, que hayan sido categorizados ${ }^{5}$ en los niveles I y II, y que se desempeñaran como directores de becas y/o tesis.

Investigadores en formación: aquellos que estuvieran incluidos en proyectos acreditados, y que hayan sido categorizados en los niveles III y IV.

Investigadores noveles (becarios de pregrado y posgrado): aquellos que mostraran cierta trayectoria en cátedras vinculadas con la investigación, y que se hayan desempeñado en algunos de los grupos de investigación consolidados; dichos sujetos han atravesado un doble proceso de selección para eventualmente conformar equipos de investigación, el primero de carácter in formal, ya que fueron invitados por los docentes investigadores a integrar equipos. El segundo proceso de índole formal, para acceder al sistema de becas mediante la presentación a convocatorias anuales en las que sus antecedentes fueron evaluados por comisiones de investigadores.

Tesistas de licenciatura: aquellos sujetos que se encontraban desarrollando su tesina de graduación sin vínculo formal con dichos equipos, sin relación laboral con ellos y conectados exclusivamente con el director del equipo a través de la dirección de su tesis.
En el presente trabajo se construyeron categorías nucleadas en función de los tópicos señalados con anterioridad y otras a posteriori elaboradas en terreno, partiendo del trabajo de campo realizado y en función de los marcos teóricos escogidos.

\section{Discusión de Resultados}

Organizamos los resultados y sus discusiones de modo integral, para lo cual optamos por realizarlo según los territorios formativos para la investigación disciplinar en biología.

\section{El campo, territorio donde el sujeto se somete el objeto real El campo es un territorio propicio para el descubrimiento}

El campo es el territorio por excelencia del biólogo. Es en él donde la empiria se presenta en su forma más plena, donde la teoría parece quedar rezagada en pos de la exaltación de los sentidos. El campo estructura una relación sujeto/objeto laxa, negociada, abierta. Es allí donde el objeto es elocuente o esquivo, donde el sujeto se muestra extraño a su objeto o inquisidor con él. Es allí donde el descubrir se hace patente y donde el sujeto se advierte ante el mundo como constructor de conocimiento.

Buscamos la teoría, pero también buscamos la relación $[\ldots]$ es como un ida y vuelta, cuando vos encontrás algo decís ¿será nuevo? [...] Si vos sabés que hay algo interesante, observemos más, pero siempre estás relacionando con lo que ya conociste $[\ldots]$ vas elaborando (Directora-Paleontología $(\mathrm{P}))$.

Al encontrar el diente ya te imaginás cómo era el bicho, vas elaborando hasta donde llegó ese mar, vas imaginando situaciones paralelas que no es solamente la observación, la experiencia, el

\footnotetext{
${ }^{4}$ En todos perfiles aludidos se ha entrevistado a un sujeto por equipo.

${ }^{5}$ En Argentina, los docentes investigadores, a través de la Secretaria de Políticas Universitarias, son categorizados en niveles comprendidos entre I y V en orden decreciente, de acuerdo con la trayectoria en la investigación, docencia y gestión universitaria.
} 
conocimiento, el interés, yo creo que se juegan un montón de valores (Directora- P).

El trabajo de campo constituye el espacio de encuentro entre el deber ser o lo probable y la realidad. Es el lugar donde se ponen en contraste las categorías teóricas y empíricas, donde se retoca la teoría por la contundencia de la empiria o donde se redibuja la realidad; finalmente, donde se ajusta el dato ideal con el real.

Sergio estudia coleópteros, al principio íbamos cualquier día, pero después él fue viendo que en luna nueva no salen sus bichos, entonces tiene que ir otro día, eso te va enseñando el campo, entonces empezamos a ver cuáles eran los factores (Investigador en formación-Antropología (A)).

En mis trampas empecé usando unas que son tubos de tela mosquitera y abajo el cebo, las moscas entraban, pero comían el cebo, se me pudrían [...] modifiqué esto, y lo que hago ahora es poner en un vasito, y le pongo un tul arriba, entonces el olor las atrae pero no comen [...] Con la experiencia en el campo uno va viendo qué es mejor (Becario de posgrado-A).

\section{El campo: el territorio donde priman los procesos observacionales}

Cobra relevancia introducir aquí la observación, ya que demanda un proceso complejo y sistemático en dónde la categorización y la percepción hacen parte de su estructura cognoscitiva. Así, el observar interroga a lo observado desde los capitales epistemológicos obtenidos, para profundizar en su reconocimiento y comprensión. Al observar, no es el ojo el que lo hace sino el sujeto construido socialmente por vivencias y acciones educativas diversas y simultáneas.

Durante el proceso de observación, el análisis no sólo permite la ubicación y delimitación del objeto de estudio, también implica la comparación y una confrontación primaria entre la teoría y la realidad.
En el proceso de observación, la objetividad no radica sólo en el registro puntual y exacto de los datos observados, sino que implica un conocimiento suficiente del campo cultural y cognoscitivo del observador al enfrentarse con su objeto de estudio. Si bien la objetividad no está ajena a la fidelidad pretendida, ésta se sitúa en una comunidad, en una historia y en un mundo que permiten otorgar un sentimiento de realidad a lo observado.

En el hecho de observar, ya estás haciendo investigación porque en el momento vos ya estás relacionando con lo ya conocido (Directora-P).

Uno cuando va al campo, va con ideas preconcebidas, ya tenés mucha evidencia paleontológica previa, geológica, y vos vas mirando y hay cosas que yo me doy cuenta después en el laboratorio que la observación esta por ahí focalizada por los preconceptos porque vas viendo si es cierto o no (Investigador en formación-P).

\section{El campo: territorio habilitador de procesos reflexivos e imaginativos}

Moreno Lache et al. (2011) sostienen que este territorio se presenta como una práctica espacial y discursiva; es un momento dialógico entre y con los actores. Se trata de un posicionamiento ontológico, epistemológico y metodológico respecto a cómo se concibe a la realidad y a la relación sujeto-objeto.

Es un proceso de tensión y construcción de conocimiento en donde se articulan y dialogan los saberes, donde se construye un espacio con base en las experiencias de uno y de los otros. Así, el campo atraviesa al mismo investigador, es el mismo campo quien sitúa al investigador para realizar situaciones de campo, porque las decisiones del investigador no se encuentran en un vacío, ellas están atravesadas por las praxis construidas socio-históricamente:

Uno no es cien por ciento objetivo, tenés una serie de preconceptos, de teorías, que vos fuiste elaborando 
y que dirige la observación [...] pensar que la ciencia es una actividad objetiva es ilusorio, porque a la ciencia la generan los seres humanos que traen ideas preconcebidas, que viven en un contexto histórico, cultural, social determinado (Directora-P).

\section{El trabajo de campo: territorio anhelado de los biólogos}

Dos integrantes del equipo de paleontología (becaria de pre grado y tesista de licenciatura), no realizaron trabajo de campo, dado que se encontraban cursando la carrera de grado, la cual exige presencia en las asignaturas.

En la siguiente cita, destacamos la necesaria presencia y acompañamiento de un experto que pueda guiar a un novel en los modos de desempeño en el campo:

Si bien tendría que haber ido al campo, tenía muchos ejemplares para trabajar, por eso ir al campo lo restringimos. Yo quiero hacer los primeros viajes con mi director, porque no sé cómo tomar la orientación, Claudia me explicó por correo cómo tomar la orientación, por eso necesito ir con él para ver (Tesista de licenciatura-P).

En contraposición presentamos el ejemplo de una tesista que enfatiza el valor del trabajo de campo en donde se producen los aprendizajes vivenciales en terreno:

Es en el campo donde se dan las situaciones con la naturaleza y donde uno aplica todo lo que aprende [...] Yo me acuerdo de cosas de primer año que vimos en el campo, vos te vas al campo y sabes identificar qué planta es [...] es porque lo viviste (Tesista de licenciatura-A).

\section{El campo...un territorio exponente de la corporeidad del investigador}

El campo es el territorio agreste, el que se impone al investigador, el que pone condiciones sobre las cuales no se discute, el que aun sin tener fronteras netamente delimitadas, define claramente lo atinente a su espacio. Así, el biólogo requerirá de la solidaridad de lo corporal para la realización de un buen trabajo de campo

El trabajo de campo es sacrificado, ahí te das cuenta si te va a gustar o no [...] más allá de las incomodidades, días de mal tiempo, comer lo que venga más rápido, de mucho esfuerzo [...] ves que a la larga vale la pena $[\ldots]$ Es una cosa rara, porque lo que a uno le apasiona, es lo que hace que uno pueda aguantar esas cosas en el camino (Becaria de pregrado-A).

\section{Las reconfiguraciones desde las praxis cotidianas hacia las praxis de índole científica: continuidades y rupturas}

Asumimos que la praxis del investigador no se construye en un vacío, ya que se vale de vivencias anteriores. En este caso se observa cómo un investigador, sin ser biólogo, tenía desempeño en el campo, lo cual contribuyó a desarrollarse en este territorio en el que continúa construyendo experiencias de un modo más específico y orientado a las particularidades de su objeto de investigación:

Tengo experiencia en el campo, porque me crié en San Miguel en el campo, entonces voy a un monte de noche y me ubico bien, y me ayudó en esto de buscar y saber dónde están los bichos, de haber conocido bastante los bosques, salí de esos lugares $[\ldots]$ ahora me estoy dando cuenta en qué mes encuentro los bichos que me sirven para determinar esta especie, entonces voy aprendiendo [...] vamos aprendiendo (Investigador en formación-A).

Presentamos el ejemplo de una tesista de licenciatura, quien se encontraba transitando las primeras experiencias de campo, donde la dificultad era más notoria dado que no estaba adaptada al contexto y los requerimientos que el campo impone: 
Se me complicó con el tiempo, porque venía a la mañana y al mediodía, imaginate el calor, dos veces al día me iba y venía, y tenés que estar un buen rato ahí, no haces todo así nomás y te vas, y así fue una semana, siete días fue dos veces al día todos los días, ya no daba más (Tesista de licenciatura-A).

\section{El campo: territorio identitario y definitorio de los biólogos}

La configuración identitaria del biólogo se materializa en el campo, ya que es el espacio real en donde el investigador se vincula directamente con el objeto y despliega todas las habilidades aprendidas durante su formación:

El biólogo tiene que aprender a estar sucio, de campamento, le tiene que gustar la naturaleza, y el que no sabe observar no puede ser biólogo desde ningún punto de vista, creo que es la base (Directora-P).

\section{El campo: espacios de incertidumbre e imprevisibilidad}

Si bien la planificación de campo se halla normada, la concreción de dicho plan queda supeditado a aquello que ese territorio habilita. Así, el clima, la hidrografía, el suelo, la fauna, la flora impondrán sus leyes, a las que no habrá otro remedio que acatarla, so pena de desbaratar la investigación. Así, en este territorio la contingencia reina y lo previsible suele desmoronarse, es el lugar donde el sujeto de ciencia queda a merced de lo imponderable:

Me robaron la jaula, porque tiene la estructura de madera y justo hicieron asado y la ocuparon, a la cabeza me la dejaron por suerte. Conseguí otra jaula del laboratorio, porque tenía que seguir mirando, y ésa ya no me la robaron porque era de hierro (Tesista de licenciatura-A).

Otras dificultades, es el mal tiempo, a todo el mundo le pasa eso con sus muestreos, que no es predecible el tema de la lluvia (Tesista de licenciatura-A).

\section{El campo...territorio donde la emoción tiene lugar}

En este territorio se presentan aspectos de índole emotivo, ya sean en el hallazgo de los objetos, en las vicisitudes imprevistas, en los descubrimientos efectuados:

La primera vez que me fui a buscar un fósil, cuando lo encontré, fue la emoción de vida y aprendí a ver cómo se encontraban y encontré un montón (Directora-P).

\section{El aspecto socializador del campo}

Por otra parte, en este territorio se ponen en juego cuestiones actitudinales, se desarrollan valores de tolerancia, respeto, cooperación y convivencia. Los tiempos y dinámicas son distintas en el territorio del campo. Generalmente son largas horas de trabajo intenso fuera de los cercos institucionales, lo cual habilita cierta desconexión de las obligaciones cotidianas diarias, y el desarrollo pleno de los lazos interpersonales, las subjetividades y las conductas "no científicas".

En equipo es más entretenido, yo voy, cuelgo mi trampa y me vuelvo, pero si estaba con alguien colgaba mis trampas, pegaba una recorrida, y podría encontrar otro bicho que me sirva, pero al estar solo estás con otros tiempos, o te puede pasar algo y nadie se entera (Becario de posgrado-A).

Lo que hago, me dio la posibilidad de tener experiencias científicas y culturales [...] Sacamos un fósil en La Puna, y tuvimos que aprendernos rituales, porque en el mundo quechua vos no podes extraer algo, estás ofendiendo a la Pachamama, entonces hicimos los rituales y luego sacamos el fósil (Investigador en formación-P).

\section{El laboratorio, territorio donde el objeto real se somete al sujeto}

El laboratorio se presenta como un espacio artificial creado para fines científicos. Así, a diferencia del 
campo, el investigador dominará y someterá a su objeto a sus dispositivos y protocolos habituales. En este sentido, el objeto de estudio obtenido en el campo sufrirá una nueva reconfiguración, de un modo más racional y asequible.

\section{El laboratorio, un territorio para describir analizar y comparar en pos de la justificación del conocimiento}

El biólogo en el laboratorio, a diferencia del campo donde lo emocional tiene lugar, hace uso de una racionalidad expresa. Se vuelve sutil, analítico, preciso; opera muy protocolar y pautadamente; desnuda al objeto de su contexto y lo pone a su merced.

El laboratorio es el imperio de la técnica, la que abre la jugada a las descripciones, análisis y comparaciones que el biólogo pone en juego:

El material se trae a laboratorio y se van viendo las muestras bajo la lupa, se les toman fotografía, si tiene restos se retira con una aguja histológica. Una vez realizado esto, y con un buen conocimiento previo de lo que son todos los tipos de estructuras, uno ya más o menos puede saber si es una estructura nueva y puede hacer una descripción de la estructura (Becario de posgrado-P).

\section{El laboratorio: un territorio para nuevos descubrimientos}

Si bien el contexto de laboratorio es el espacio privilegiado para la justificación del conocimiento, no es menos cierto que la novedad puede tener algún protagonismo. Así, cuando falta información, el laboratorio puede ser un territorio para experimentar, manipular, o incidir sobre los hechos en pos de la construcción de nuevo conocimiento.

Encontramos un bicho raro en el Perichón y me dicen los de Buenos Aires que sería interesante poder saber de qué se alimenta, entonces fui probando, como sé que son carnívoros por teoría, les fui ofreciendo cosas y no le encontraba la vuelta $[\ldots]$ como en teoría sabemos que pueden comerse entre ellos le ofrecí una araña más chica y automáticamente la caza, entonces entender que estas arañas se alimentan de otras más chicas era todo un descubrimiento (Investigador en formación-A).

Cuando fuimos a La Puna estaba seguro, por los estudios geológicos, que estábamos trabajando en afloramientos modernos, entonces todos los bichos que extraíamos los asignaba a especies modernas. Cuando vine al laboratorio me di cuenta que mi observación estaba sesgada. Después de un análisis exhaustivo de laboratorio, sabemos que son faunas viejas, que tienen cuatro millones de años (Investigador en formación-P).

\section{Descubrir en el laboratorio puede implicar emociones}

Lo emotivo e intuitivo, aunque en menor medida que el campo, también se encuentra presente en este territorio, lo que se expresa cuando el investigador "habla" con sus objetos o discute acerca de éstos con sus colegas. Expresan alegrías cuando aquellos elementos difusos encontrados en el campo adquieren cierta forma a través de sistemas categoriales teóricos que darán fundamento a ese objeto.

Revisando las claves, te encontrás con la sorpresa de las cosas nuevas [...] a mí me gusta mucho lo nuevo [...] Cuando encontraba un género desconocido me parecía híper interesante (Becario de pregrado-A).

\section{El laboratorio: configurador de hábitos de los investigadores, lugar de teorías encarnadas y de entrenamiento de los sentidos}

En el territorio del laboratorio el investigador desarrolla una serie de métodos y técnicas para acercarse a su objeto. Dichos modos de acercamiento precisan de un saber experiencial particular, los cuales a medida que el investigador construye su praxis, lo internaliza y naturaliza, realizando luego ciertas prácticas de modo mecánico: 
Me dedicaba a mirar bichos, a veces algunos me llevaban dos meses poder saber qué eran, y hoy miro uno y sé hasta qué especie es, lo que antes me costaba mucho (Investigador en formación-A).

Cuando empecé a trabajar con material estaba todo el tiempo consultando bibliografía. Ahora ese tiempo lo tengo ahorrado, porque uno ya mira con mucho bagaje del tema específico y se hace casi automático (Becario de posgrado-P).

\section{Los límites que nos imponen las regulaciones académicas}

Emergieron aspectos que refieren a casos que se descubren en el laboratorio pero no pueden comunicarse porque no pueden respaldarse científicamente. Aquí se evidencia la fuerte normatividad de la disciplina y por sobre todo de la comunidad científica como reguladora de las actividades académicas que realizan los investigadores en sus respectivas disciplinas, pues es ésta quien establece criterios de aceptación en cuestiones metodológicas o conceptuales. Así, los descubrimientos del laboratorio se hacen con un ojo puesto en la potencial crítica o aceptación (Knorr Cetina, 2005).

Hay una tesis que nunca la publicaron porque se cree que está mal, pero mundialmente el tema todos lo tocamos sin jugarnos del todo. Es embromado jugarse porque no hay algo actual que sea igual [...] es pura investigación y mucha imaginación en cuanto a cómo era, porqué la inundación movía el terreno, pero nadie quiere arriesgarse, no hay bases firmes (Directora-P).

\section{Laboratorio: territorio reconfigurador de las praxis educativas}

En el laboratorio, las praxis de investigaciones anteriores también afectan a las selecciones subsiguientes, modelando a su vez las bases de las futuras decisiones. Así, el investigador construirá a través de las distintas experiencias nuevos aprendizajes, considerando lo aprendido anteriormente y reconfigurando los conocimientos actuales ante cada situación presentada, diferenciando las situaciones de laboratorio en carácter de alumno y en carácter de novel investigador:

En el laboratorio aprendés las estructuras generales, pero no te hacen hacer distinciones como alumno, que después si uno trabaja en eso, en etología, tiene que aprender la técnica, que lo va aprendiendo en realidad cuando ya está trabajando, investigando [...] Para acercarte a la práctica tenés que acercarte a un grupo y empezar a investigar [...] no llegas sabiendo (Becario de pregrado-A).

\section{El escritorio, territorio donde el sujeto define, construye y gestiona el objeto simbólico (el objeto narrado)}

A este territorio lo entendemos como el ámbito donde se habilitan condiciones de tiempo, lugar y disposición para el desarrollo de aspectos sustanciales del quehacer científico. Por ello, organizamos este apartado en tres secciones; las dos primeras aluden a procesos de escritura científica: una con el de gestionar y otra con la intención de comunicar. La última sección refiere a cuestiones interpersonales que abonan al buen funcionamiento del equipo.

\section{El objeto narrado para gestionar}

Decidimos revalorizar el aspecto de la gestión en este territorio, dado que aquí se sustancian las cuestiones referentes a la planificación de proyectos en pos de la continuidad de la investigación en curso e investigaciones futuras, gestión y programación del trabajo de campo, pedido de recursos, realización de notas, elaboración de informes, entre otros.

Los aspectos mencionados con anterioridad, podrían considerarse inocuos y hasta incluso rutinarios para el equipo, no obstante, enfatizamos la importancia de incluirlo como una de las actividades estructurantes del proceso de investigación ya que forma parte de la "gestión del objeto". 
Gestionar el objeto de investigación, más allá de los aspectos técnicos, implica también preconfigurar el objeto a través del discurso, dotarlo de estatus epistemológico, asignarle relevancia para abordarlo bajo los cánones disciplinares, es decir, persuadir a los pares acerca de la importancia de estudiarlo en ese marco disciplinar:

Yo inicié proyectos de investigación propios en el 99, no es fácil hacer un proyecto, si bien sabía hacer porque yo había participado [...] buscar bibliografias, pensar un tema, justificar es lo más difícil, porque uno quiere hacer porque le gusta, pero tiene que buscar una justificación científica (Directora-A). Para armar el proyecto tenemos mucha participación todos ¿`cómo vamos a muestrear? ¿Dónde? ¿Qué? En ese aspecto hacemos todos juntos, discutimos bastante $[\ldots]$ Hasta que nos pongamos de acuerdo (Investigador en formación-A).

Si en la primera instancia se preconfigura el objeto, en la segunda se informa acerca del objeto reconstruido. La primera alude a las determinaciones ideales, a lo posible o necesario, a lo que debiera ser, a lo prescripto por la norma. Lo segundo refiere a los condicionamientos reales, a lo dado, a lo que es, a lo que pudo expresarse de la norma.

\section{El objeto narrado para comunicar}

Narrar para comunicar demanda acciones distintas de la explicitada en el contexto anterior. Este proceso se propone narrar para una comunidad científica que avale los aportes realizados en la investigación llevada a cabo. Aquí se atiende a una nueva reconfiguración del objeto: la presentación del objeto de investigación de manera narrada y argumentada. Las razones que dirigen nuestra atención a la escritura académica disciplinar es que consideramos que en esta práctica se dan los procesos donde los descubrimientos deben ser justificados, donde las decisiones deben ser aún mucho más "fundamentadas", donde la objetivación del conocimiento queda a merced de la buena retórica y uso de recursos del investigador.

Ellos cuando empiezan ya tienen que presentar algo, y ahí es que tenés que ayudarlo, qué es lo que tiene para presentar en forma inicial [...] pero sobre todo el seguimiento mío es ver que estén leyendo, que te consulten (Directora-A).

Empecé a leer otros trabajos que hagan algo parecido, y en base a eso estructuré mi trabajo porque en principio uno no sabe cómo tiene que escribir, entonces lo estructura, y hoy me doy cuenta que no hay otra forma, tiene que ser así (Becario de posgrado-A).

\section{Consideraciones generales de la escritura disciplinar en biología}

El artículo científico: un documento que esconde mucho más de lo que explicita

El artículo se presenta como un producto final, no obstante, para convertirse en un manuscrito científico deberá transitar una conversión, desde el razonamiento donde el investigador exhibe sus preocupaciones con pureza salvaje hasta la narración, donde estos razonadores parecen cambiar de fe, tornándose fieles a las scripturas (escrituras de autoridad), más que a las preocupaciones a partir de las cuales se originó la investigación, (Knorr Cettina, 2005).

En lugar de un relato de las negociaciones sociales de los investigadores nos encontramos con un registro selectivo de los resultados transitorios de esas negociaciones, permeado por la particularización técnica (Knorr Gettina, 2005).

Una anécdota, a mí me encanta mucho leer, entonces los primeros papers eran muy floridos, con muchas descripciones, adjetivos. Un día me agarró el director de la revista más importante de la Argentina en paleontología, yo era pichón, y le digo [...] ¿qué tal mi papers? [...] con una timidez y me dice está bien pero vos escribís demasiado florido, y 
esta disciplina es taca, taca, son ideas puntuales y sin vueltas (Investigador en formación-P).

Para Bernstein (1999) se trata de un discurso vertical caracterizado por estructuras discursivas más sistemáticas, jerárquicas y explícitas que el discurso horizontal de las ciencias sociales. Así, el discurso identitario refleja valores asociados y constituye lo que Morales y Cassany (2008) denominan comunidades discursivas según los géneros científicos.

Peredo Merlo (2016) ubica a las ciencias naturales dentro de las disciplinas convergentes, las cuales suelen mantener ideologías y valores comunes en los juicios de calidad que emiten y que pueden observarse en los productos evaluados por comités de expertos reconocidos, donde su principal preocupación radica en el mantenimiento de estándares de control intelectual. En palabras de Bar (2005), el autor de un artículo con contenido biológico se asume como relator y como evaluador objetivo, como alguien que se mantiene ajeno a la realidad.

\section{Aspectos estratégicos que se ponen en juego en la escritura científica}

Escribir para una comunidad científica implica desplegar ciertos modos de estrategias para que el trabajo trascienda. Cabe destacar aquí que los investigadores deben dar cuenta ante los organismos financiadores de todo lo producido, para lograr la continuidad y permanencia en la investigación. En el campo de la biología, la evaluación de la productividad se sostiene sobre criterios cualitativos y cuantitativos; el primero dado por las publicaciones en revistas de alto impacto, y el segundo por la cantidad de artículos elaborados

La política de CONICET es elitista, todos saben que tienen que publicar en revistas de alto impacto, eso se determina por citabilidad, hay empresas que se dedican a eso, entonces si los trabajos publicados en tal revista están cien veces citados esas tienen más impacto que una revista cuyos trabajos pocas veces son citados (Directora-A).

En el campo disciplinar de los biólogos, las revistas de alto impacto generalmente se encuentran en el idioma inglés, de allí que la escritura en este idioma se convirtió en tradición disciplinar instalada:

CONICET tiene en cuenta el índice de citabilidad, y un trabajo va a ser más citado si está en inglés; entonces termina siendo necesario que uno publique en inglés para aumentar su citabilidad (Becario de posgrado-P).

En nuestra disciplina, los papers tienen que salir en inglés [...] es un idioma todavía más duro y más concreto, el problema es que uno nunca termina escribiendo lo que realmente quiere, porque son ideas que te nacen en la cabeza en español y que realmente están desarrolladas así (Investigador en formación-P).

\section{Tensiones expresadas en la escritura}

La estructura de los artículos esconde tensiones entre posturas propias a sostener y perspectivas ajenas; entre lo que se descubre y lo que se justifica; entre la teoría y la empiria; entre el ser y el deber ser; entre lo sugerido por el director y lo redactado por el tesista; entre lo hecho y lo comunicable.

$\mathrm{Si}$ bien el investigador debe tener autonomía intelectual, debe reconocer que atrás de ésta hay una suma de voces académicas previas que es necesario identificar y distinguir de la propia, si se quiere ser reconocido por una comunidad científica (Peredo Merlo, 2016).

Tengo un libro que tiene muchas justificaciones de determinados estudios [...] entonces sirve para la introducción. Una vez que pasas esta etapa y tenés noción de lo que estás haciendo ya te va saliendo solo, porque en la introducción vos fundamentas con el conocimiento que tenés [...] ya sabes lo que 
estás haciendo, entonces redactas en eso (Becario de pregrado-A).

Por otra parte, las recomendaciones para la escritura disciplinar del director a sus tesistas residen particularmente en las bibliografías utilizadas y en la metodología, aspectos que dan sustancia y justificación al trabajo realizado:

Ya con lo metodológico una vez que arrancan siguen solos, no es que tenés que decirle a cada rato mira tenés que hacer este método, esto otro (Directora-A).

Vos no podés hacer ANOVA si no probás que tus datos pueden aplicarse a eso, entonces tuve que hacer dos test más para ver si se ajustaban mis datos, y sí se ajustaban, por suerte, o sino tenía que aplicar otro test de vuelta (Becario de pregrado-A).

En cuanto a la sección resultados, los biólogos lo caracterizan como descriptivo, como algo que deviene naturalmente, sin advertir que los resultados son siempre emergentes de determinadas selecciones metódicas. Lo que se obtiene no es independiente de cómo es el método (Knorr Cetina, 2005).

Los resultados tenés que contar lo que obtuviste nada más, obtuve tantos números en ambiente tanto, los índices estadísticos me dieron estos valores, vas contando nada más los datos (Becario de pregrado-A).

Las secciones de discusión y conclusiones encierran complejidad, dado que es aquí donde deben contrastar con los demás autores, diferenciar sus resultados respecto de los otros. Este apartado muestra los debates en torno de lo que se descubre y de las justificaciones, de los aportes propios y el de los otros referentes.

Aquí la lógica escritural cambia de sentido, ya que deben expresarse en argumentos que sumen adherentes. En esta construcción argumentativa es donde el investigador no puede evadir la asunción de una posición. Así, si bien la valoración de la evidencia empírica es superlativa para la biología, no es menos cierto que ciertos rasgos propios del discurso obrante en el texto pueden coadyuvar a reafirmarla (Bar, 2011: 105).

La discusión a mí me costaba, porque hay que fundamentar tus resultados, comparar para qué sirven, qué aportás, hoy me es más fácil armar; ahora estuvimos armando un paper con un becario y trato de enseñarle eso (Investigador en formación-A).

En las discusiones es donde uno se da cuenta que puede generar algo nuevo (Becario de posgrado-P).

La discusión no es sólo una comparación, es una discusión entre mis resultados con los de otros, y porqué mis resultados son distintos a los de él, la parte gruesa en donde se ve la experiencia del investigador es en la discusión (Becario de pregrado-A).

Knnor Cetina (2005) expresa que la validación es en la práctica un proceso de formación racional de consenso en la comunidad científica, que los validadores que conforman esa comunidad "son independientes" de los productores del conocimiento, y que su juicio crítico constituye una base objetiva de validación. No obstante, la comunicación de lo descubierto, sus decisiones y sus selecciones se organizan conforme con las respuestas esperadas de determinados miembros de la academia. Es la propia comunidad científica la que como respuesta a un nuevo aporte de conocimiento le asigna o no peso al descubrimiento.

Siempre hay casos en donde uno trata de acomodar los hechos al marco teórico. Siempre me decía un paleontólogo famoso [...] vos tenés que mostrar lo que te conviene, no lo que contradice; las observaciones que contradicen tu hipótesis vos no las muestres [...] y mucha gente hace eso (Investigador en formación-P). 
La cita anterior pone en discusión la supuesta objetividad de los investigadores, pues se advierte una posición dogmática y sesgada, dado que éstos buscarán pruebas que lo validen, poniendo los datos al servicio de sus convicciones. Caso contrario es el que presentamos a continuación, el que muestra cierta apertura a la novedad, apertura a los nuevos conocimientos, aun cuando esto implique la autorefutación.

Sí refuto, hasta me autorefuté y en el marco de trabajo lo digo que me equivoqué. Ameghino siempre decía $[. .$.$] cambiaré tantas veces de opinión como$ la evidencia me lo diga [...] no es malo cambiar de opinión, lo malo es quedarse en una postura (Investigador en formación-P).

\section{Vínculos interpersonales}

En referencia con los rasgos implícitos, incluimos a los aspectos motivacionales y emocionales, los cuales influyen en el buen funcionamiento del equipo y el trabajo de la investigación. Es aquí donde se expresan aspectos de reconocimiento, valores que no refieren exclusivamente a la ciencia pero que contribuyen al quehacer del grupo, a su posterior mejora, a su consolidación en la producción del conocimiento.

Los valores son fundamentales, la responsabilidad en todos los actos de la vida, el respeto a tu colega y a trabajar en grupo [...] permanecemos como grupo hace más de treinta años [...] eso creo que es el gran valor y el mérito (Directora-P).

\section{Las jornadas científicas, territorios donde el sujeto comunica el objeto narrado (objeto simbólico)}

Los quehaceres científicos se comunican a través de espacios instituidos en la academia, tales como revistas, congresos jornadas, conferencias (Moreno Bayardo, 2015). Dichos espacios comportan dos modalidades, uno de carácter formal y otro de índole más informal.
En cuanto a los aspectos formales, refieren explícitamente a la socialización del conocimiento, en donde se habilita la reconstrucción de las ideas a partir de los intercambios entre los investigadores.

En el caso de los artículos lo hacen a través de nuevas publicaciones y en los congresos a través del contacto personal. En los dos casos se confrontarán las tensiones entre "autoridades académicas", y entre el descubrimiento y la justificación de los conocimientos. No obstante este territorio se presenta como el espacio propicio para la defensa de la postura del investigador:

En un congreso el equipo presentó los trabajos de vinchucas que estábamos haciendo en Corrientes y alguien de Buenos Aires dijo: "Ustedes se van a pasear al campo [...]" La directora del congreso le saltó, porque el que no fue al campo a buscar vinchucas no puede abrir la boca (Directora-A).

La gente de vertebrados me cuestionaban que tenía las trampas muy cerca, porque las separo por 70 metros, y ellos que hacen aves me dicen que lo hacen cada 200 metros, pero yo les dije si vos comparás lo que vuela una mosca con un pájaro, no es lógico, y que no tienen el mismo trato un vertebrado que un invertebrado para hacer un análisis de diversidad (Becario de posgrado-A).

En cuanto a los aspectos informales de los congresos nos remitimos a los vínculos que se generan entre los investigadores con líneas de investigación y el intercambio entre los integrantes del mismo equipo. Dichas instancias implican interactuar, afrontar en conjunto el desafio intelectual resultante de los debates, asimilar positivamente las nuevas perspectivas con una actitud abierta al enriquecimiento interpersonal

Los intercambios te abren la cabeza [...] saber que el otro está en lo mismo [...] se me empiezan a prender las luces de lo que yo puedo hacer (Investigador en formación-A). 
Los congresos contribuyen en hacer contactos, saber que hay gente que trabaja con algo parecido, o conocerles las caras [...] Por ejemplo a mi director lo vi en una jornada, que fue después de pedir becas (Becario de posgrado-A).

\section{Entramado de los distintos territorios para la formación en investigación disciplinar}

Hemos nucleado los resultados emergentes en el siguiente cuadro, el que ilustra la dialéctica puesta en la construcción de la praxis científica del equipo de investigación.

\section{Cuadro 1. Construcción de las praxis científicas del equipo de investigación}

Comunicación del objeto tornado real

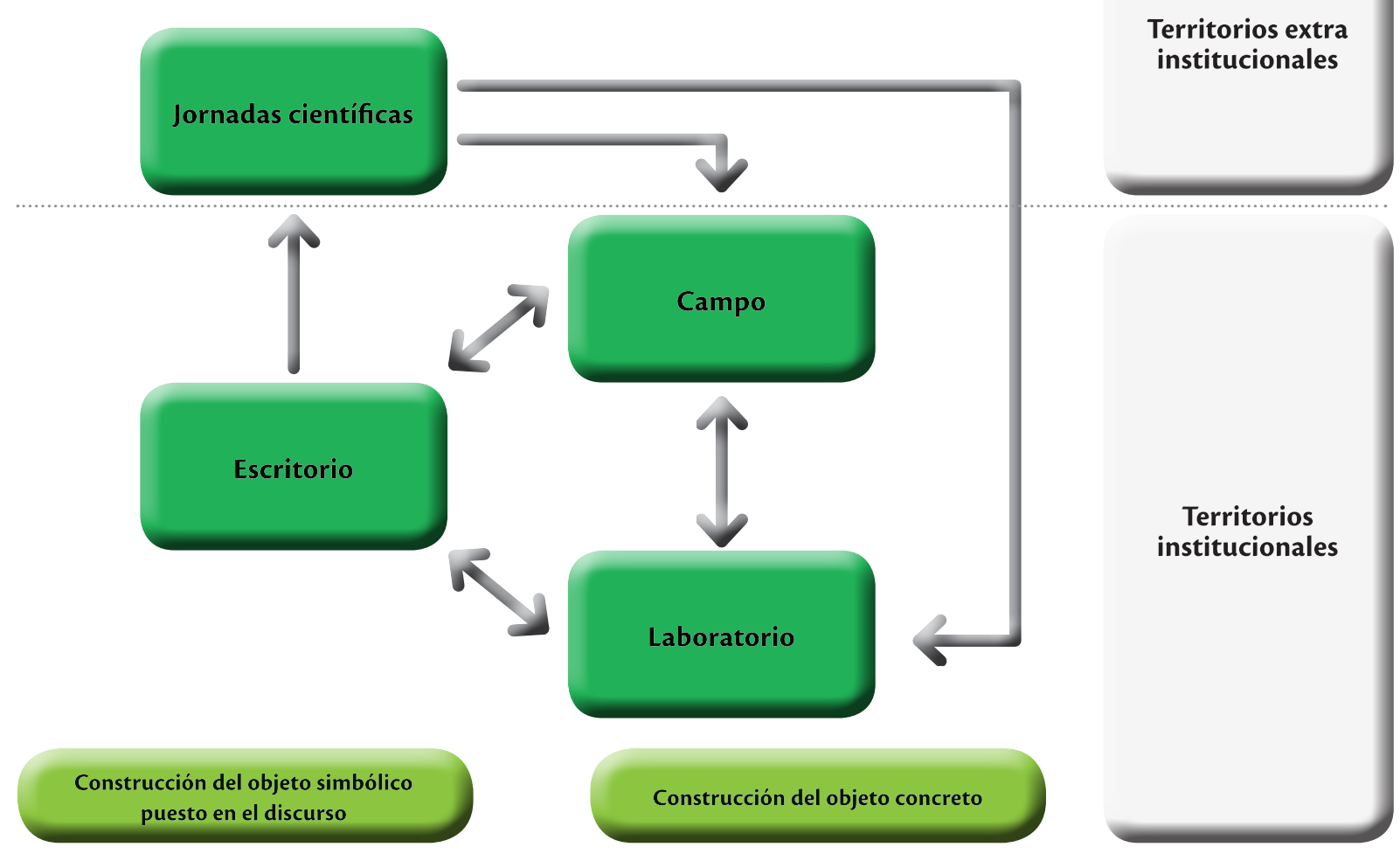

Si intentamos reelaborar la génesis o el tránsito de los investigadores en los distintos territorios, pareciera que ilusoriamente se procede a realizar una serie de pasos para el logro de la comunicación de los resultados obtenidos. En desacuerdo con ello, sostenemos que los procesos de investigación son dinámicos, recursivos, complejos, no lineales.
Formalmente, los inicios de la investigación, caracterizado por momentos de incertidumbres, preguntas, planteamiento de objetivos, se dan en el territorio formativo "escritorio", ya que es éste el locus que articula y da sustancia a los tres restantes. Es allí donde aparentemente se inician los procesos de la gestión para la investigación expresados en la 
planificación y debates de lo que se desarrollará en un futuro. Así también, en el escritorio se objetivarán los datos para luego comunicarlos a la comunidad científica.

El campo y el laboratorio se presentan como territorios significativos para la empiria, pues es en ellos donde el objeto idealizado y gestionado se torna en objeto real; libre en el primero, restringido en el segundo.

Cuando el investigador regresa al escritorio a formular los escritos, relee y revisa las prácticas del campo y del laboratorio, coteja con experiencias pasadas, realiza consultas con el equipo, resignifica la empiria y reconfigura su objeto, todo lo cual redunda en un "objeto aséptico" a comunicarse. Así, quedan ocultas las recursividades y las reconfiguraciones teóricas y metodológicas derivadas de las praxis materializadas en los tres territorios precedentes.

Sostenemos que las características disciplinares otorgadas a la biología acerca de su conservadurismo, de su monismo metodológico y de su linealidad, no son tales, ya que esto se expresa sólo en el discurso neutro propio de la tradición disciplinar. Entendemos que los modos de producción en biología no siguen ese curso, sino más bien que forman parte de un entramado complejo que se sobresimplifica al traducirse en texto, el que por cierto obedece a una lógica acordada y aprobada por la comunidad.

Esta dinámica dialéctica que hemos intentado mostrar obedece a la fluencia de los procesos que demanda el oficio de investigar, los cuales en realidad no tienen origen ni fin definido, como tampoco cursos únicos. Dichos procesos quedarán a merced de las praxis de los investigadores, quienes en cada territorio construirán y reconstruirán el objeto inicial formal en objeto final, real y comunicable.

Otro aspecto que se reconfigura en cada territorio es la relación formativa entre el director y el novel investigador. El primero lo acompañará en los modos de relación con su objeto de estudio, ya sea en campo orientándolo para su aprehensión en estado natural, a "dominarlo" en el laboratorio, a reconstruirlo en el escritorio a través del discurso, y a comunicarlo en las jornadas científicas. De este modo, en cada territorio el investigador dará condiciones al novel investigador para que la praxis educativa disciplinar construida en el contexto de educación, se reconstruya en praxis científica enmarcada en los contextos de innovación y evaluación de la ciencia. Dichas praxis están dadas en ámbitos sociohistóricos, por lo que no son neutras ni inocuas, sino cargadas de supuestos y valores que otorgan sentido al quehacer disciplinar.

Como síntesis última de lo que venimos exponiendo, afirmamos que la relación sujeto-sujeto (formado-en formación) en cada territorio formativo, viene dada por la relación sujeto-objeto en los espacios de producción y justificación del conocimiento, y que cada uno de ellos supone matices epistemológicos puestos en modos y dispositivos de formación para la investigación. Así, el maestro ofrece al aprendiz una ontología del fenómeno de la que éste deberá hacerse cargo, compromiso que supone reproducirlo en su praxis, ajustarlo conforme con nuevas situaciones; en definitiva, sostenerlo en el marco del paradigma imperante si se precia de ser un buen profesional en la materia.

El formador y el sujeto en formación se encuentran insertos en un campo disciplinar que se encarna en el proceso pedagógico. Dicho proceso viene dado por dos condicionantes, uno concreto y situado, el territorio; y otro abstracto y formal, la epistemología disciplinar. El primero habilita y limita espacialmente las operaciones materiales y simbólicas que allí se puedan construir en función de las normas explicitas e implícitas que rigen el funcionamiento en ese espacio. El segundo impone modos de acercamiento al objeto según los imperativos disciplinares, propone formas de indagación y define normas de justificación del conocimiento.

En función de lo expuesto, se expresan dos tipos de relaciones que operan simultáneamente en el 
territorio. Por un lado, la relación sujeto-objeto cristalizada en una epistemología disciplinar; por otro, el vínculo sujeto-sujeto (maestro-aprendiz) dado en la pedagogía. Así, afirmamos que la primera forma de relación determina la segunda, y que los matices o formas de expresión de lo propiamente disciplinar, incidirá fuertemente en las maneras que se sustancie el dispositivo pedagógico.

Entendemos que el atravesar territorios supone distintas configuraciones sujeto-objeto y sujeto-sujeto; que en cada una de ellas el formador entrenará a su

\section{Referencias}

Bar, Aníbal (2013), "Saberes y prácticas cognitivas en el contexto de la formación disciplinar en biología", Tesis Doctoral en Ciencias Cognitivas, Argentina, Facultad de Humanidades de la Universidad Nacional del Nordeste.

Bar, Aníbal (2011), "Argumentar y explicar en el contexto de la formación universitaria en biología", Revista Argentina de la Educación Superior, vol. 4, núm. 4, pp. 92113, <http://www.revistaraes.net/revistas/raes4_art5. pdf $>$ [Consulta: julio de 2016].

Bar, Aníbal (2005), Textos de biología y modelos de explicación. Los fenómenos biológicos desde la bibliografia universitaria y escolar, Corrientes, Moglia.

Bernstein, Basil (1999), "Vertical and horizontal discourse: an essay", British fournal of Sociology of Education, vol. 20, núm. 2, pp, 157-173, <http://lchc.ucsd.edu/mca/ Paper/JuneJuly05/BernsteinVerHor.pdf> [Consulta: agosto de 2017].

Biglan, Anthony (1973), "Relationships between subject matter characteristics and the structure and output of university departments", Gournal of Applied Psychology, vol. 57, núm. 3, pp, 204-213, <http://edshare.soton. ac.uk/15017/2/Biglan_-_1973_-_Relationships_ between_subject_matter_characteristics_and_the_ structure_and_output_of_university_departments. discípulo conforme con el sistema de reglas, epistemológicas en primera instancia y pedagógicas en segunda; y que en ese devenir el maestro intentará que el alumno construya su praxis científica a expensas de su praxis de estudiante precedente. Así, en esta última tarea el director intentará que esa construcción no se dé en cualquier dirección, sino en aquella que concluya en el modelo de ciencia aceptado por la biología y matizado por lo que el formador le ha dado, lo que en definitiva será la puesta de su sello personal en ese nuevo sujeto devenido de novel a experto.

pdf> [Consulta: mayo de 2013].

Cortina, Adela (2003), "El sentido de las profesiones", en A. Cortina y J. Conill, 10 palabras clave en la ética de las profesiones, Navarra, Editorial Verbo Divino, pp. 13-28). Echeverría, Javier (1998), Filosofia de la ciencia, Madrid, Akal.

Flores, Miriam y Aníbal Bar(2017), "Territorios formativos para la investigación en biología. La perspectiva de las directoras de equipos de investigación”, Ponencia presentada en X Jornadas de Investigación Educativa, Córdoba, Universidad Nacional de Córdoba, < https: / / rdu.unc.edu.ar/handle/11086/5255> [Consultado: octubre de 2017].

Fourtor Olliver, María (201 1), "Los grupos de investigación: un acercamiento desde una mirada ética", Revista Iberoamericana sobre Calidad, Eficacia y Cambio en Educación, vol. 9, núm. 2, pp. 106-117.

Hartley, Jean (1994), "Case studies in organizational research", en C. Casell y G. Symon (eds.), Cualitative methods in organizational research, Londres, Sage Publications, pp. 208-229.

Knorr Cetina, Karin (2005), La fabricación del conocimiento. Un ensayo sobre el carácter constructivista y contextual de la ciencia, Buenos Aires, Universidad Nacional de Quilmes. Morales, Oscar y Daniel Cassany (2008), "Leer y escribir 
en la universidad: hacia la lectura y la escritura crítica de géneros científicos", Revista Memoralia, núm. 5, pp. 69-82.

Moreno Bayardo, María (2015), "La formación para la investigación en el posgrado. Una propuesta de desarrollo de habilidades", en M. Moreno Bayardo y V. Mancovsky, La formación para la investigación en el posgrado, Buenos Aires, Noveduc, pp. 28-111.

Moreno Lache, Nubia, Liliana Rodríguez Pizzinato y Jorge Sánchez Ardilla (2011), La salida de campo... se hace escuela al andar, Bogotá, Universidad Distrital Francisco José de Caldas, <http://www.geopaideia.com/publicaciones/ salida_campo.pdf $>$ [Consulta: enero de 2017].

Perelo Merlo, María (2016), "Lectura y ciencia en diversos posgrados y disciplinas", Revista de la Educación Superior, vol. 45, núm. 180, pp. 41-54, <http://dx.doi.org/10.1016/j. resu.2016.07.001 > [Consulta: junio de 2017].

Stake, Robert (1995), The art of case study research, Londres, Sage Publications, <http://investigacionsocial.sociales. uba.ar/files/2013/03/STAKE_investigacion-conestudio-de-casos.pdf> [Consulta: mayo de 2015].

\section{Cómo citar este artículo:}

Flores, Miriam-Liset y Aníbal-Roque Bar (2019), "Los territorios de la formación para la investigación en biología", en Revista Iberoamericana de Educación Superior (RIES), México, UNAM-IISUE/Universia, vol. x, Núm. 29, pp. 67-85, DOI: https://doi.org/10.22201/ iisue.20072872e.2019.29.523 [consulta: fecha de última consulta]. 